\title{
Desenvolvimento de Ceraeochrysa cincta (Schneider, 1851)(Neuroptera: Chrysopidae) alimentada com dieta artificial na fase larval
}

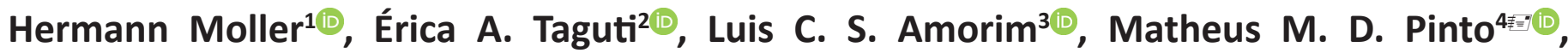 \\ Sergio A. De Bortoli ${ }^{4}$ \\ ${ }^{1}$ Universidade Estadual Paulista "Júlio de Mesquita Filho", UNESP, Laboratório de Ecologia Aplicada, Jaboticabal, São Paulo, Brasil. \\ 2Universidade Estadual Paulista "Júlio de Mesquita Filho", UNESP, Laboratório de Biossistemática de Hemiptera, Jaboticabal, São \\ Paulo, Brasil. ${ }^{3}$ Ourofino Agrociência, Guatapará, São Paulo, Brasil. "Universidade Estadual Paulista "Júlio de Mesquita Filho", UNESP, \\ Laboratório de Biologia e Criação de Insetos, Jaboticabal, São Paulo, Brasil. \\ 莑=Corresponding author: matheusmoreirafacu@gmail.com
}

\author{
Edited by: Francisco J. S. Duque
}

Received: May 21, 2020. Accepted: June 13, 2020. Published: October 30, 2020.

Development of Ceraeochrysa cincta (Schneider, 1851) (Neuroptera: Chrysopidae) fed with artificial diet in the larval phase

Abstract. Mass production of lacewings is still a bottleneck for applied biological control and for biofactories producing natural enemies, due to the difficulties of large-scale production and the cost of the undertaking and the production of their alternative prey. Thus, the objective of this work was to evaluate the viability of an alternative artificial diet to rearing of Ceraeochrysa cincta (Schneider, 1851) (Neuroptera: Chrysopidae). Three treatments were analyzed for this purpose, consisting of: 1) natural diet - eggs of Corcyra cephalonica (Stainton, 1866) (Lepidoptera: Pyralidae), 2) artificial diet honey + beer yeast (1:1) and 3) natural diet (1) + artificial diet (2), and were offered to the larval stage of the predator. The biological parameters of $C$. cincta evaluated where: duration of larval, pupal and adult phases; pupal weight; pupal viability; sex ratio; preoviposition and oviposition periods and number of eggs per female. Were obtained adults only in the treatments 1 and 3 and, in the treatment 2 no insect reached the pupal stage. The results obtained demonstrated that the artificial diet, when offered in isolation, causes significant differences in the duration of the larval period, especially in the 3rd instar, and in the weight of the pupae, with the other similar parameters in the treatments, but can serve as a nutritional supplement.

Keywords: biological control, rearing, biology.

Os crisopídeos (Neuroptera: Chrysopidae) são insetos de ampla distribuição mundial e associados a diversos cultivos e pragas (Freitas 2001). Ceraeochrysa Adams, 1982 (Neuroptera: Chrysopidae), o segundo gênero mais diverso dentre os Chrysopidae, agrupa cerca de 60 espécies (Freitas et al. 2009; Sosa \& Freitas 2010), das quais 33 são registradas no Brasil (Martins \& Machado 2020), algumas espécies apresentam importantes atributos predatórios. Freitas \& Penny (2001) realizaram estudos em ecossistemas agrícolas brasileiros e coletaram 15 espécies de Ceraeochrysa com ocorrência para diferentes culturas.

As larvas desta família atuam vorazmente contra diversos artrópodes fitófagos como pulgões, ácaros, lepidópteros, cigarrinhas, tripes e moscas-branca, o que os tornam excelentes agentes de controle biológico (New 1975; Canard \& Principi 1984).

O conhecimento e domínio de uma metodologia de multiplicação desses insetos em laboratório para liberações em campo, de forma que se produza quantidade e qualidade suficientes para esse fim, ainda é um grande desafio para que o uso desses predadores em áreas de manejo integrado de pragas seja uma realidade. Além disso, informações sobre o desenvolvimento desse predador alimentado com diferentes presas em sistemas agrícolas e a associação com outros métodos de controle são ainda relativamente escassas. É de extrema importância a determinação de parâmetros de desenvolvimento em diferentes sistemas de criação, com variações bióticas e abióticas, e seus reflexos no desenvolvimento desses predadores, tais variações podem causar mudanças diferentes de acordo com a espécie estudada (Pessoa 2009; Chapman et al. 2002).
Pessoa et al. (2010) relataram que a criação massal e eficiente de crisopídeos depende de alguns fatores, tais como: técnicas de manipulação nas diferentes fases de desenvolvimento do inseto, da dieta oferecida e dos custos de produção e adaptabilidade. Vários dos aspectos biológicos observados nos organismos são respostas ao tipo ou modo de alimentação que Ihes foi ofertado ao longo de seu desenvolvimento e o conhecimento sobre nutrição de insetos assume cada vez mais importância, em virtude da necessidade crescente de produção massal de insetos, particularmente de inimigos naturais, quesito necessário para a utilização no manejo integrado de pragas.

Vários estudos relataram que larvas de algumas espécies de crisopídeos são mantidas, com boa eficiência, com fontes de alimento obtidas de outras criações de insetos, entre os quais ovos de lepidópteros, como a traça-dos-cereais, Sitotroga cerealella (Olivier, 1769) (Lepidoptera: Gelechiidae), e a traça-da-farinha, Anagasta kueniella (Zeller, 1879) (Lepidoptera: Pyralidae) (Figueira et al. 2002; Carvalho \& Souza 2009; De Bortoli \& Murata 2011). O acréscimo de novas informações dos ciclos biológicos e comportamentos de espécies de crisopídeos poderão facilitar a disponibilidade desses predadores para uso no controle biológico aplicado (Nuñez 1988).

Assim, o objetivo deste estudo foi avaliar a efetividade de um alimento alternativo para a para criação em laboratório de $C$. cincta.

As atividades dos bioensaios foram desenvolvidas no Laboratório de Biologia e Criação de Insetos (LBCl) do Departamento de Fitossanidade (DEF) da Faculdade de Ciências Agrárias e Veterinárias da Universidade Estadual Paulista (FCAV/Unesp), Campus de Jaboticabal, SP, em 
condições controladas de temperatura ( $25 \pm 20 \mathrm{C})$, umidade relativa ( $75 \pm 10 \%$ ) e de luminosidade ( $12 \mathrm{~h}$ de luz).

Os bioensaios foram desenvolvidos com insetos oriundos da criação estoque do $\mathrm{LBCl}$. Os insetos da criação recebem como alimento ovos de $C$. cephalonica na fase larval e à base de mel e levedo de cerveja, na proporção 1:1, na fase adulta.

Os bioensaios foram realizados com 300 ovos de C. cincta, 100 ovos para cada tratamento, de mesma idade (menos de 24 horas da oviposição), individualizados em tubos de ensaio de fundo chato $(8,5$ $\mathrm{cm}$ de altura $X 2,5 \mathrm{~cm}$ de diâmetro). Após a eclosão, as larvas foram alimentadas com ovos de $C$. cephalonica durante o primeiro ínstar. Atingido o segundo ínstar, foram utilizadas 60 larvas, sendo essas as repetições para os diferentes tratamentos: 1 ) dieta padrão $-4,0 \mathrm{mg}$ de ovos de $C$. cephalonica; 2 ) dieta artificial - $1,0 \mathrm{mg}$ de mel e levedo de cerveja na proporção 1:1 e 3) dieta com 2,0 mg de ovos de $C$. cephalonica e 0,5 mg de mel e levedo de cerveja na proporção 1:1. As quantidades de dietas foram estabelecidas com base em pré-testes quando foi observado a quantidade consumida pelas larvas durante 24 horas; as dietas foram oferecidas diariamente até a fase pupal. A dieta artificial foi oferecida em pedaços de espumas fixadas na extremidade de tubos plásticos contendo água para manter a sua umidade, e a dieta padrão despejada dentro dos tubos com o auxílio de um pincel.

Os tubos de vidro contendo os insetos de cada tratamento foram verificados diariamente para acompanhar o desenvolvimento, oferecimento das dietas e realizar a manutenção e limpeza dos recipientes.

As pupas obtidas foram mantidas nos tubos de vidro até a emergência dos adultos. Após a emergência dos adultos (machos e fêmeas) de cada tratamento, foram colocados 6 casais de cada tratamento em gaiolas (tubos de PVC $10 \mathrm{~cm}$ de diâmetro X $20 \mathrm{~cm}$ de altura), cobertas com tecido tipo "voile".Os casais foram alimentados com solução de mel e levedo de cerveja (1:1), oferecida na parte superior das gaiolas, juntamente com água.

Ao longo do desenvolvimento dos insetos foram determinados os seguintes parâmetros biológicos: duração das fases larval e pupal; longevidade de adultos; peso de pupas; viabilidade de pupas; razão sexual; períodos de pré-oviposição e de oviposição, e número de ovos por fêmea.

O delineamento utilizado foi o inteiramente casualizado (DIC), com os resultados submetidos aos testes de Bartlett para constatação de homocedasticidade (PROC GLM) e de Cramer von Mises para normalidade (PROC UNIVARIATE). Os dados de duração do 2ㅇ e 3o ínstares e os períodos de pré-oviposição e de oviposição não apresentaram as pressuposições da ANOVA. Então, foi conduzida a análise não paramétrica (PROC NPAR1WAY) e as médias comparadas pelo teste de Kruskal Wallis $(P>0,05)$. Os dados de duração do período pupal, peso da pupa, longevidade de fêmeas e de machos e número de ovos por fêmea atenderam as pressuposições da ANOVA e as médias comparadas pelo teste de Tukey $(P>0,05)$. Todas as análises foram conduzidas utilizando o software SAS (SAS Institute 2015).

A dieta artificial junto com os ovos de C. cephalonica (Dieta 3) oferecida foi capaz de fornecer condições às larvas de $C$. cincta continuar seu desenvolvimento, com exceção de quando a dieta foi oferecida de forma isolada (Dieta 2), quando as larvas alimentadas apenas com a solução de mel e levedo de cerveja atingiram somente o $3^{\circ}$ ínstar e não foram capazes de passar da fase larval para a pupal.

Todos os tratamentos apresentaram alta sobrevivência, acima de $80 \%$, para as larvas de segundo ínstar de $C$. cincta. No entanto, para as larvas de terceiro ínstar, apenas a Dieta 2 proporcionou o mesmo desempenho, enquanto que as dietas 1 e 3 promoveram sobrevivência de $63,33 \%$ e $70,00 \%$, respectivamente. As larvas alimentas com as dietas 1 e 3 apresentaram taxas de pupação de $68,42 \%$ e $80,77 \%$ com viabilidade de $56,41 \%$, e $54,76 \%$, respectivamente. Nenhum indivíduo alimentado com a Dieta 2 atingiu a fase pupal.

A duração do segundo ínstar larval de $C$. cincta foi influenciada pelas dietas oferecidas $\left(\chi^{2}=45,24 ; G L=2 ; P<0,0001\right)$, foi maior quando as larvas foram alimentadas apenas com ovos de $C$. cephalonica (Tab. 1). Os valores obtidos foram menores por larvas alimentadas com ovos de três espécies de lepidópteros (De Bortoli et al. 2009), e com ovos e ninfas de Bemisia tabaci (Gennadius, 1889) (Hemiptera: Aleyrodidae), biótipo B (Auad et al. 2001).

A duração do terceiro ínstar $\left(\chi^{2}=33,95 ; G L=2 ; P<0,0001\right)$ também foi afetada significativamente pelos tratamentos; as larvas alimentadas com a Dieta 2 tiveram esse período, em média, 3,60 vezes mais longo que as larvas alimentadas com a Dieta 1, e 2,62 vezes maior que daquelas alimentadas com a Dieta 3 (Tab. 1). Não há relatos de resultados semelhantes a este na literatura, que registrassem uma duração do terceiro ínstar tão prolongada.

$O$ peso da pupa $(F=22,66 ; P<0,0001)$ também foi afetado significativamente pelas dietas, as pupas obtidas a partir das larvas alimentadas com a Dieta 3 pesaram, em média, 1,52 vezes mais que daquelas alimentadas com a Dieta 1. Porém, a duração do período pupal não foi influenciada pelos tratamentos $(F=1,08 ; P=0,3055)$ (Tab. 1). Pessoa et al. (2010) obtiveram peso de pupa semelhante ao obtido neste trabalho com a Dieta 3 quando alimentaram larvas de C. cincta com ovos de Diatraea saccharalis (Fabricius, 1794) (Lepidoptera: Crambidae) e S. cerealella $(7,1 \pm 0,02 \mathrm{mg}$ e 7,0 $\pm 0,03 \mathrm{mg}$, respectivamente).

Table 1. Duração dos períodos imaturos (2ํ́nstar até pupa) e peso de pupas de Ceraeochrysa cincta (Schneider, 1851) (Neuroptera: Chrysopidae) alimentada com diferentes dietas.

\begin{tabular}{lcccc}
\hline Tratamentos & $\begin{array}{c}\text { 20 ínstar } \\
\text { (dias) }\end{array}$ & $\begin{array}{c}\text { 30 ínstar } \\
\text { (dias) }\end{array}$ & Pupa (dias) & PP (mg) \\
\hline Dieta 1 & $3,71 \pm 0,07 \mathrm{a}^{1}$ & $4,050,21 \pm \mathrm{b}^{1}$ & $12,14 \pm 0,15 \mathrm{a}^{2}$ & $4,98 \pm 0,37 \mathrm{~b}^{2}$ \\
Dieta 2 & $3,12 \pm 0,06 \mathrm{~b}$ & $14,59 \pm 1,52 \mathrm{a}$ & - & - \\
Dieta 3 & $3,23 \pm 0,08 \mathrm{~b}$ & $5,57 \pm 0,23 \mathrm{c}$ & $11,91 \pm 0,15 \mathrm{a}$ & $7,58 \pm 0,40 \mathrm{a}$ \\
\hline
\end{tabular}

${ }^{1}$ Médias \pm erro padrão seguidas da mesma letra na coluna não diferem pelo teste de Kruskal Wallis $(P>0,05) .{ }^{2}$ Médias \pm erro padrão seguidas da mesma letra na coluna não diferem pelo teste de Tukey $(\mathrm{P}>0,05)$. $\mathrm{PP}-\mathrm{Peso}$ de Pupa. Dieta 1 - padrão à base de ovos de C. cephalonica $(4,0 \mathrm{mg})$. Dieta 2 - artificial à base de mel + levedo de cerveja - 1:1 (1,0 mg). Dieta 3 - padrão à base de ovos de $C$. cephalonica $(2,0 \mathrm{mg})+$ dieta artificial à base de mel + levedo de cerveja - 1:1 (0,5 mg).

As longevidades das fêmeas $(F=0,02 ; P=0,9019)$ e dos machos de C. cincta $(F=0,95 ; P=0,3553)$ não apresentaram diferença significativa (Tab. 2). De Bortoli et al. (2009), obtiveram fêmeas e machos mais longevos $(72,20 \pm 15,26$ dias e $68,70 \pm 10,04$ dias, respectivamente) quando alimentaram larvas de $C$. cincta com lagartas e ovos dos lepidópteros $A$. kuehniella e S. cerealella.

Table 2. Longevidade dos adultos (dias), períodos de pré-oviposição e oviposição (dias) e número de ovos por fêmea de Ceraeochrysa cincta (Schneider, 1851) (Neuroptera: Chrysopidae) alimentada com diferentes dietas.

\begin{tabular}{lccccc}
\hline Tratamentos & $\begin{array}{c}\text { Longevidade } \\
\text { Fêmeas }\end{array}$ & $\begin{array}{c}\text { Longevidade } \\
\text { Machos }\end{array}$ & $\begin{array}{c}\text { Período } \\
\text { de pré- } \\
\text { oviposição }\end{array}$ & $\begin{array}{c}\text { Período de } \\
\text { oviposição }\end{array}$ & $\begin{array}{c}\text { Número } \\
\text { de ovos }\end{array}$ \\
\hline Dieta 1 & $28,00 \pm 9,04 \mathrm{a}^{2}$ & $27,33 \pm 6,76 \mathrm{a}^{2}$ & $12,75 \pm 0,25 \mathrm{a}^{1}$ & $15,50 \pm 2,86 \mathrm{a}^{1}$ & $\begin{array}{c}143,00 \pm 49,27 \\
\mathrm{a}^{2}\end{array}$ \\
\hline
\end{tabular}

Dieta 2

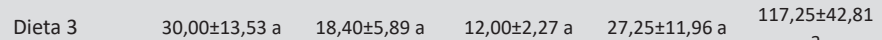

${ }^{1}$ Médias \pm erro padrão seguidas da mesma letra na coluna não diferem pelo teste de Kruskal Wallis $(P>0,05) .{ }^{2}$ Médias \pm erro padrão seguidas da mesma letra na coluna não diferem pelo teste de Tukey $(P>0,05)$. Dieta $1-$ padrão à base de ovos de $C$. cephalonica $(4,0$ $\mathrm{mg})$. Dieta 2 - artificial à base de mel + levedo de cerveja - 1:1 (1,0 mg). Dieta 3 - padrão à base de ovos de $C$. cephalonica $(2,0 \mathrm{mg})+$ dieta artificial à base de mel + levedo de cerveja $-1: 1(0,5 \mathrm{mg})$

Os períodos de pré-oviposição $\left(\chi^{2}=1,0854 ; \mathrm{GL}=1 ; \mathrm{P}=0,2975\right)$ e de oviposição $\left(\chi^{2}=1,7287 ; G L=1 ; P=0,1886\right)$ de $C$. cincta também não diferiram estatisticamente nas diferentes dietas (Tab. 2). De Bortoli et al. (2009) também não constataram diferença significativa quando analisaram esses parâmetros com as larvas de $C$. cincta alimentadas com ovos de diferentes espécies de lepidópteros. No entanto, os valores médios obtidos por aqueles autores para o período de pré-oviposição foram menores, com variação entre $4,20 \pm 0,63$ dias e 4,70 $\pm 0,48$ dias, enquanto que o de oviposição foi mais longo $(52,50 \pm 10,28$ dias e $58,80 \pm 10,59$ dias) que os encontrados neste trabalho, para préoviposição e para oviposição, respectivamente. Por outro lado, Nuñez (1988) obteve duração média bem maior para o período de pré- 


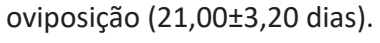

O número de ovos por fêmea de $C$. $\operatorname{cincta~}(F=0,16 ; P=0,7068)$ não apresentou diferença entre as dietas avaliadas (Tab. 2) e foi inferior aos valores encontrados por De Bortoli et al. (2009). Oliveira et al. (2016), ao alimentaram larvas Ceraeochrysa cubana (Hagen, 1861) com ovos de $S$. cerealella obtiveram, também, valores médios superiores $(476,2 \pm 3,21$ ovos), entretanto quando alimentaram larvas desse predador com ninfas do 1 o ao 40 ínstar da mosca negra dos citrus, Aleurocanthus woglumi Ashby, 1915 (Hemiptera: Aleyrodidae), o número de ovos foi menor, $54,2 \pm 12,88$. Toda essa variação entre diferentes estudos explorando diferentes fontes do alimento, demonstra o quanto os parâmetros biológicos desses insetos podem variar de acordo com a origem e/ou qualidade de alimento que recebem, que de forma geral, podem ser de diversas espécies, porém, com a utilização de ovos de lepidópteros na maioria das dietas padrões de crisopídeos (Carvalho \& Souza 2009).

Tanto a nutrição quantitativa quanto a qualitativa de dieta e de presas afetam o estágio imaturo de desenvolvimento dos insetos e, para os crisopídeos, os parâmetros de duração, peso e sobrevivência das fases larval e pupal têm relação direta com as necessidades nutricionais dos indivíduos (Canard \& Principi 1984). E o incremento da dieta natural de predadores com alimentos alternativos tem demonstrado ser boa estratégia para o aprimoramento do controle biológico (van Rijn et al. 2002; Janssen \& Sabelis 2015) tanto para condições de campo quanto em laboratório.

Recursos como pólen, néctar, exsudatos, honeydew e inclusive floema podem fazer parte da alimentação de diversos artrópodes predadores e muitas das vezes estas fontes de alimentos são determinantes para a conservação de uma população de predadores no ambiente (Barbosa \& Wratten 1998; Coll \& Guershon 2002). Os benefícios de uma variedade maior de alimentos de outras origens que não a de presas, podem surtir efeitos inclusive em parâmetros não avaliados nesse trabalho, como por exemplo, capacidade de busca por presas (McEwen et al. 1993; Limburg \& Rosenheim 2000).

Provavelmente as larvas de $C$. cincta alimentadas somente com a dieta artificial a base de mel e levedo de cerveja não obtiveram os nutrientes necessários para o desenvolvimento normal e atingirem o estágio de pupa. O maior peso de pupas dos indivíduos alimentados com a Dieta 3 deve ter sido resultado de carboidratos presentes na composição da dieta artificial, que deve ter funcionado como complemento alimentar.

Os resultados obtidos nesse estudo indicaram que a dieta artificial composta por mel e levedo de cerveja na proporção de 1:1, quando oferecida de forma exclusiva, não se mostrou efetiva como alimento alternativo para larvas de $C$. cincta na criação em laboratório. Entretanto, a dieta artificial apresentou bom potencial como complemento/ suplemento nutricional adicionado a alimentação natural, o que pode ser interessante em eventual escassez da dieta principal, em criações massais desta espécie, visto que nesse caso, a quantidade de alimento natural pode ser reduzida pela metade.

\section{Agradecimentos}

Ao programa de Agronomia (Entomologia Agrícola) da Universidade Estadual Paulista "Júlio de Mesquita Filho", Unesp Campus Jaboticabal, a CAPES e ao CNPq por fornecer todos os suportes estruturais e fomento necessários para a realização do experimento.

\section{Contribuições dos Autores}

Elaboração do projeto e tutoria: Pinto, M. M. D.; De Bortoli, S. A. Revisão do projeto: De Bortoli, S. A. Condução dos bioensaios e redação do manuscrito: Moller, H.; Taguti, E. A.; Amorim, L. C. S.; Pinto, M. M. D. Revisão do manuscrito: De Bortoli, S. A.; Pinto, M. M. D.

\section{Referências}

Auad, A. M.; Toscano, L. C.; Boiça Júnior, A. L.; Freitas, S. (2001) Aspectos biológicos dos estádios imaturos de Chrysoperla externa (Hagen) e Ceraeochrysa cincta (Schneider) (Neuroptera: Chrysopidae) alimentados com ovos e ninfas de Bemisia tabaci (Gennadius) Biótipo B (Hemiptera: Aleyrodidae). Neotropical Entomology, 30(3): 429-432. doi: 10.1590/S1519-566X2001000300015

Barbosa, P.; Wratten, S. D. (1998) Influence of plants on invertebrate predators: implications to conservation biological control. In: Barbosa, P. (Ed.) Conservation biological control. pp.83-100. Academic Press.

Canard, M.; Principi, M. M. (1984) Feeding habits. In: Canard, M.; Seméria, Y.; New T. R. (Eds.) Biology of Chrysopidae. pp.27-76. Springer Netherlands.

Carvalho, C. F.; Souza, B. (2009) Métodos de criação e produção de crisopídeos. In: Bueno, V. H. P. (Eds.). Controle biológico de pragas: produção massal e controle de qualidade. pp.91-92. Lavras: UFLA.

Chapman, J. W.; Reynolds, D. R.; Smith, A. D.; Riley, J. R.; Pedgley, D. E.; Woiwod, I. P. (2002) High altitude migration of the diamondback moth Plutella xylostella to the UK: a study using radar, aerial netting, and ground trapping. Ecological Entomology, 27(6): 641650. doi: 10.1046/j.1365-2311.2002.00472.x

Coll, M.; Guershon, M. (2002) Omnivory in terrestrial arthropods: mixing plant and prey diets. Annual review of entomology, 47(1): 267-297. doi: 10.1146/annurev.ento.47.091201.145209

De Bortoli, S. A.; Murata, A. T.; Brito, C. H.; Narciso, R. S. (2009) Aspectos biológicos de Ceraeochrysa cincta (Neuroptera: Chrysopidae), em condições de laboratório. Revista de Biologia e Ciência da Terra, 9(2): 101-106.

De Bortoli, S. A.; Murata, A. T. (2011) Morphometrical aspects of Ceraeochrysa paraguaria (Navás, 1920) (Neuroptera: Chrysopidae) feeding on different preys. Comunicata Scientiae, 2(2): 122-125. doi: $10.14295 /$ cs.v2i2.19

Figueira, L. K.; Carvalho, C. F.; Souza, B. (2002) Influência da temperatura sobre alguns aspectos biológicos de Chrysoperla externa (Hagen, 1861) (Neuroptera: Chrysopidae) alimentada com ovos de Alabama argillacea (Hübner, 1818) (Lepidoptera: Noctuidae). Ciência e Agrotecnologia, 26: 1439-1450.

Freitas, S. (2001) Criação de crisopídeos (Bicho-lixeiro) em laboratório. Jaboticabal: Funep.

Freitas, S.; Penny, N. D. (2001) The green lacewings (Neuroptera: Chrysopidae) of Brazilian agro-ecossystems. Proceedings of the California Academy of Science, 52: 245-395.

Freitas, S.; Penny, N. D.; Adams, P. A. (2009) A revision of the New world genus Ceraeochrysa (Neuroptera: Chrysopidae). Proceedings of the California Academy of Sciences, 60(15): 503.

Janssen, A.; Sabelis, M. W. (2015) Alternative food and biological control by generalist predatory mites: the case of Amblyseius swirski. Experimental and Applied Acarology, 65: 413-418. doi: 10.1007/s10493-015-9901-8

Limburg, D. D.; Rosenheim, J. A. (2001) Extrafloral nectar consumption and its influence on survival and development of an omnivorous predator, larval Chrysoperla plorabunda (Neuroptera: Chrysopidae). Environmental Entomology, 30(3): 595-604. doi: 10.1603/0046225X-30.3.595

Martins, C. C.; Machado, R. J. P. (2020) Chrysopidae. In: Catálogo Taxonômico da Fauna do Brasil. http://fauna.jbrj.gov.br/fauna/ faunadobrasil/96. Access on: 15.ii.2020.

McEwen, P. K.; Jervis, M. A.; Kidd, N. A. C. (1993) Influence of artificial honeydew on larval development and survival in Chrysoperla carnea [Neur., Chrysopidae]. Entomophaga, 38(2): 241-244. doi: 10.1007/BF02372559

New, T. R. (1975) The biology of Chrysopidae and Hemerobiidae (Neuroptera), with reference to their usage as biocontrol agents: a review. Transactions of the Entomological Society of London. 127: 115-140. doi: 10.1111/j.1365-2311.1975.tb00561.x

Nuñez, E. (1988) Ciclo biológico y crianza de Chrysoperla externa y Ceraeochrysa cincta (Neuroptera, Chrysopidae). Revista Peruana de Entomología, 31: 76-82.

Oliveira, R.; de Oliveira Barbosa, V.; Vieira, D. L.; De Oliveira, F. Q.; de Luna Batista, J.; De Brito, C. H. (2016) Development and reproduction of Ceraeochrysa cubana (Neuroptera: Chrysopidae) 
fed with Aleurocanthus woglumi (Hemiptera: Aleyrodidae). Semina: Ciências Agrárias, 37(1): 17-23.

Pessoa, L. G. A.; Freitas, S.; Loureiro, E. S. (2010) Adequação de dietas para criação de adultos de Chrysoperla externa (Hagen) (Neuroptera: Chrysopidae). Arquivos do Instituto Biológico, 77(4): 723-725.

Pessoa, L. G. A. (2009) Efeito da variação da temperatura sobre o desenvolvimento embrionário e pós-embrionário de Chrysoperla raimundoi Freitas \& Penny (Neuroptera: Chrysopidae). Arquivos do Instituto Biológico, 76: 239-244.

SAS Institute Inc. (2015) SAS/IML ${ }^{\circledR} 14.1 v U s e r ' s$ Guide. Cary, NC: SAS Institute Inc. https://www.sas.com/pt_br/home.html.

Sosa, F.; Freitas, S. (2010) New Neotropical species of Ceraeochrysa Adams (Neuroptera: Chrysopidae). Zootaxa, 2562(1): 57-65. doi: 10.11646/zootaxa.2562.1.4

Van Rijn, P. C.; Van Houten, Y. M.; Sabelis, M. W. (2002) How plants benefit from providing food to predators even when it is also edible to herbivores. Ecology, 83(10): 2664-2679. doi: 10.1890/0012-9658(2002)083[2664:HPBFPF]2.0.CO;2 